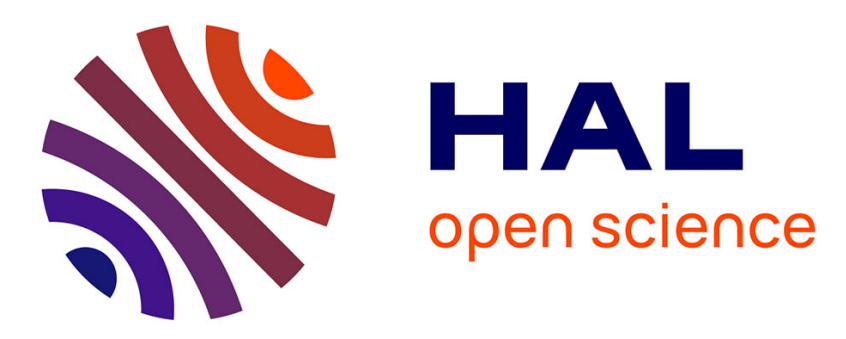

\title{
Nonlinear observer based sensor fault tolerant control for nonlinear systems
}

Dalil Ichalal, Benoît Marx, Didier Maquin, José Ragot

\section{To cite this version:}

Dalil Ichalal, Benoît Marx, Didier Maquin, José Ragot. Nonlinear observer based sensor fault tolerant control for nonlinear systems. 8th IFAC Symposium on Fault Detection, Supervision and Safety of Technical Processes, SAFEPROCESS 2012, Aug 2012, Mexico City, Mexico. pp.CDROM. hal00684702

\section{HAL Id: hal-00684702 \\ https://hal.science/hal-00684702}

Submitted on 8 Apr 2014

HAL is a multi-disciplinary open access archive for the deposit and dissemination of scientific research documents, whether they are published or not. The documents may come from teaching and research institutions in France or abroad, or from public or private research centers.
L'archive ouverte pluridisciplinaire HAL, est destinée au dépôt et à la diffusion de documents scientifiques de niveau recherche, publiés ou non, émanant des établissements d'enseignement et de recherche français ou étrangers, des laboratoires publics ou privés. 


\title{
Nonlinear observer based sensor fault tolerant control for nonlinear systems
}

\author{
Dalil Ichalal* Benoît Marx** Didier Maquin** José Ragot** \\ * IBISC Laboratory, Evry-Val d'Essonne University, \\ 40, rue de Pelvoux, Courcouronnes 91020 Evry Cedex \\ e-mail: dalil.ichalal@ibisc.univ-evry.fr \\ ** CRAN, UMR 7039, Université de Lorraine - CNRS \\ 2, avenue de la forêt de Haye, 54016 Vandouvre-lès-Nancy Cedex \\ e-mail: \{benoit.marx, didier.maquin, jose.ragot\}@univ-lorraine.fr
}

\begin{abstract}
This paper deals with the problem of sensor fault tolerant control for Takagi-Sugeno nonlinear systems. Firstly, a residual generator is designed in order to detect and isolate sensor faults. Secondly, a nonlinear observer based controller, adopting the so-called parallel distributed compensation structure is designed. This controller is based on a weighted blending of the estimated states provided by different observers. Each observer is constructed to estimate the system state from the inputs and only one output. The blending of the estimates depends on the residual magnitudes in order to minimize the use of faulty estimates in the control law. The stability of the global closed-loop system is studied by Lyapunov theory and the gains of the fault tolerant controller are obtained by solving Linear Matrix Inequalities.
\end{abstract}

Keywords: Fault-tolerant control, sensor fault, nonlinear systems, Takagi-Sugeno model

\section{INTRODUCTION}

Diagnosis is a key point in system supervision and human or process safety. An occurring fault must not only be detected and isolated, but also accommodated by a socalled fault tolerant control law, to preserve the stability and the performances of the system.

Since many years, linear models have been largely studied and many results have been obtained in the fields of fault diagnosis and fault tolerant control (Ding, 2008). However, the linearity assumption is only verified around a single operating point. In order to consider a large operating range of the system, it is important to take into account the nonlinearities in the modeling task. The obtained models are more accurate but are obviously also harder to deal with. Indeed, due to the complexity of nonlinear systems, there is no general framework. Consequently, it leads to work on specific model classes (e.g. Lipschitz systems, bilinear systems, etc).

Among the several classes of nonlinear systems, the Takagi-Sugeno (T-S) structure, introduced in (Takagi and Sugeno, 1985), is interesting since it is a "universal approximator". Any nonlinear behavior can be then approximated with a given accuracy with a T-S model (Tanaka and Wang, 2001). A T-S model is made up of a set of linear submodels and an interpolation mechanism between these submodels based on nonlinear weighting functions. A T-S model can be established using three main principal methods: linearization around a set of operating points, identification (Gasso et al., 2002) and the sector nonlinearity transformation (Tanaka and Wang, 2001).
Stability analysis and stabilization of nonlinear T-S systems are studied in (Tanaka and Wang, 2001; Guerra et al., 2006; Kruzewski et al., 2008). One can cite the use of the Lyapunov theory and the formulation of the stability conditions in terms of linear matrix inequalities (LMI). More recently the nonquadratic approaches have been developed in (Tanaka et al., 2003) in order to reduce the conservatism of the quadratic approaches. The observer design applied to state and unknown input estimation is treated in (Bergsten et al., 2002; Akhenak et al., 2008; Ichalal et al., 2009a). T-S observers-based fault diagnosis is explored in (Chen and Saif, 2007; Marx et al., 2007; Akhenak et al., 2008; Ichalal et al., 2009a). In the context of fault tolerant control (FTC), state trajectory tracking is proposed in (Ichalal et al., 2010) for actuator faults. Observer bank based controllers with switching mechanism for sensor faults is also presented in (Oudghiri et al., 2008).

In this paper, a new approach of the FTC of T-S systems is proposed. It is based on a bank of observers and a controller using a new state vector formed by a blending of all the estimated states provided by each observer. Assuming that the system states are observable from each output. Then each observer estimates the state of the system from only one output and its input. Then if a fault affects a given sensor, the controller mainly uses the estimated states provided by the other observers. This is obtained by using the residual signals to compute the blending of the estimated states. Finally, the FTC is represented by a mixture of all the local estimated states and if a sensor fault is isolated, the corresponding estimated state is disabled and the FTC becomes a mixture of the estimated states obtained from fault free sensors. 
Notations.The symbol $*$ stands for the terms induced by symmetry. The terms $0_{n}$ and $I_{n}$ define, respectively, the null square matrix and the identity matrix with dimension $n$. The non square null matrix is defined by $0_{n p \times n}$ with dimension $n p \times n$. The block diagonal matrix with $M_{1}, \ldots$, $M_{n}$ on its diagonal entries is denoted $\operatorname{diag}\left(M_{1}, \ldots, M_{n}\right)$.

Lemma 1. For any matrices $X$ and $Y$ with appropriate dimensions and a symmetric positive definite matrix $\Lambda$, the following holds

$$
X^{T} Y+Y^{T} X \leq X^{T} \Lambda^{-1} X+Y^{T} \Lambda Y
$$

Lemma 2. (Congruence lemma) Consider two matrices $X$ and $Y$. If $X$ is positive definite and $Y$ is full column rank then $Y X Y^{T}$ is positive definite.

Lemma 3. Consider a symmetric negative definite matrix $\Pi$, a matrix $X$ and a scalar $\eta$, the following hods

$$
\begin{aligned}
& \left(X+\eta \Pi^{-1}\right)^{T} \Pi\left(X+\eta \Pi^{-1}\right) \leq 0 \\
\Leftrightarrow & X^{T} \Pi X \leq-\eta\left(X+X^{T}\right)-\eta^{2} \Pi^{-1}
\end{aligned}
$$

\section{TAKAGI-SUGENO MODELING}

A given a nonlinear system $(\dot{x}(t)=f(x(t), u(t))$ and $y(t)=g(x(t)))$ can be written under the following T-S form:

$$
\left\{\begin{array}{l}
\dot{x}(t)=\sum_{i=1}^{r} \mu_{i}(\xi(t))\left(A_{i} x(t)+B_{i} u(t)\right) \\
y(t)=\sum_{i=1}^{r} \mu_{i}(\xi(t)) C_{i} x(t)
\end{array}\right.
$$

where $x(t) \in \mathbb{R}^{n}$ is the state vector, $u(t) \in \mathbb{R}^{m}$ is the control input and $y(t) \in \mathbb{R}^{p}$ represents the system output vector and where $A_{i} \in \mathbb{R}^{n \times n}, B_{i} \in \mathbb{R}^{n \times m}, C_{i} \in \mathbb{R}^{p \times n}$. The integer $r$ represents the number of submodels. The weighing functions $\mu_{i}$ are nonlinear and depend on the decision variable $\xi(t)$ which can be measurable like $u(t)$ or $y(t)$ or not measurable like the state of the system $x(t)$. In some situations (hybrid or LPV systems for example) it can also be an external signal. The weighting functions satisfy the convex sum property described by

$$
\sum_{i=1}^{r} \mu_{i}(\xi(t))=1,0 \leq \mu_{i}(\xi(t)) \leq 1, \quad \forall t, \forall i=1, \ldots, r
$$

\section{FAULT TOLERANT CONTROL DESIGN FOR T-S SYSTEMS}

\subsection{Sensor fault detection and isolation}

In the purpose of sensor fault diagnosis, the approach given in (Ichalal et al., 2009b) is adopted. In order to isolate the sensor faults, a residual vector is generated such that its $i^{\text {th }}$ component is only sensitive to the $i^{\text {th }}$ fault. Then, for a faulty system described by

$$
\left\{\begin{array}{l}
\dot{x}(t)=\sum_{i=1}^{r} \mu_{i}(\xi(t))\left(A_{i} x(t)+B_{i} u(t)\right) \\
y(t)=\sum_{i=1}^{r} \mu_{i}(\xi(t))\left(C_{i} x(t)+G_{i} f(t)\right)
\end{array}\right.
$$

where $f(t) \in \mathbb{R}^{p}$ denotes the sensor fault vector, the following residual generator is proposed

$$
\left\{\begin{array}{l}
\dot{\hat{x}}(t)=\sum_{i=1}^{r} \mu_{i}(\xi(t))\left(A_{i} \hat{x}(t)+B_{i} u(t)+L_{i}(y(t)-\hat{y}(t))\right) \\
\hat{y}(t)=\sum_{i=1}^{r} \mu_{i}(\xi(t)) C_{i} \hat{x}(t) \\
r(t)=M(y(t)-\hat{y}(t))
\end{array}\right.
$$

A filter $W_{\text {ref }}(s)$ defined by

$$
W_{\text {ref }}(s)=\left(\begin{array}{l|l}
A_{\text {ref }} & B_{\text {ref }} \\
\hline C_{\text {ref }} & D_{\text {ref }}
\end{array}\right)
$$

is introduced to model the desired response of the residual $r(t)$ to the fault $f(t)$. The design of the residual generator aims at minimizing the difference between $R_{\text {ref }}(s)=$ $W_{\text {ref }}(s) F(s)$ and $R(s)$ by determining an adequate matrix $M$. This difference can be quantified by the $\mathcal{L}_{2}$-gain from $f(t)$ to $\tilde{r}(t)=r_{r e f}(t)-r(t)$. If $W_{r e f}(s)$ is diagonal, each residual $r_{i}(t)$ is made sensitive only to the fault affecting the $i^{\text {th }}$ output. Consequently, not only fault detection but also isolation is ensured. The definition of $W_{\text {ref }}(s)$ can take benefits from an a priori knowledge on the frequency content of the fault. This additional filter must satisfy the condition $\sigma_{\min }\left(W_{\text {ref }}(s)\right) \geq 1$ where $\sigma_{\min }\left(W_{\text {ref }}(s)\right)$ represents the lowest singular value of the transfer function $W_{\text {ref }}(s)$. This constraint is made in order to avoid fault attenuation.

The design of the gain matrices of the residual generator $M$ and $L_{i}$ is performed via the optimization problem given in the theorem 4.

Theorem 4. (Ichalal et al., 2009b) The robust residual generator (5) exists if there exists symmetric and positive definite matrices $P_{1}$ and $P_{2}$, matrices $K_{i}$ and $M$ and a positive scalar $\gamma$ solving the following optimization problem

$$
\min _{P_{1}, P_{2}, K_{i}, M, \gamma} \gamma
$$

under the following LMI constraints

$$
\begin{cases}X_{i i}<0, & i=1, \ldots, r \\ \frac{2}{r-1} X_{i i}+X_{i j}+X_{j i}<0, & i, j=1, \ldots, r, i \neq j\end{cases}
$$

where, for $(i, j) \in\{1, \ldots, r\}, X_{i j}$ and $\Psi_{i j}$ are defined by

$$
X_{i j}=\left(\begin{array}{cccc}
\Psi_{i j} & 0 & -K_{i} G_{j} & C_{i}^{T} M^{T} \\
* & A_{r e f}^{T} P_{2}+P_{2} A_{r e f} & P_{2} B_{r e f} & -C_{r e f} \\
* & * & -\gamma I & G_{i}^{T} M^{T}-D_{r e f}^{T} \\
* & * & * & -\gamma I
\end{array}\right)
$$

$\Psi_{i j}=A_{i}^{T} P_{1}+P_{1} A_{i}-C_{j}^{T} K_{i}^{T}-K_{i} C_{j}$

The residual generator gains are given by $L_{i}=P_{1}^{-1} K_{i}$ and $M$. The attenuation level from the faults $f(t)$ to the virtual residual $\tilde{r}(t)=r_{r e f}(t)-r(t)$ is given by $\gamma$.

The proof is omitted, but can be found in (Ichalal et al., $2009 b)$.

\subsection{Fault tolerant control}

In order to achieve the fault tolerant control, an observer bank is used. The $k^{t h}$ observer is fed with the input of the system $u(t)$ and the $k^{t h}$ output $y^{k}(t)$ as illustrated by the figure 1 . Then, this observer can estimate fault-free states even if faults occur on the other sensors. 


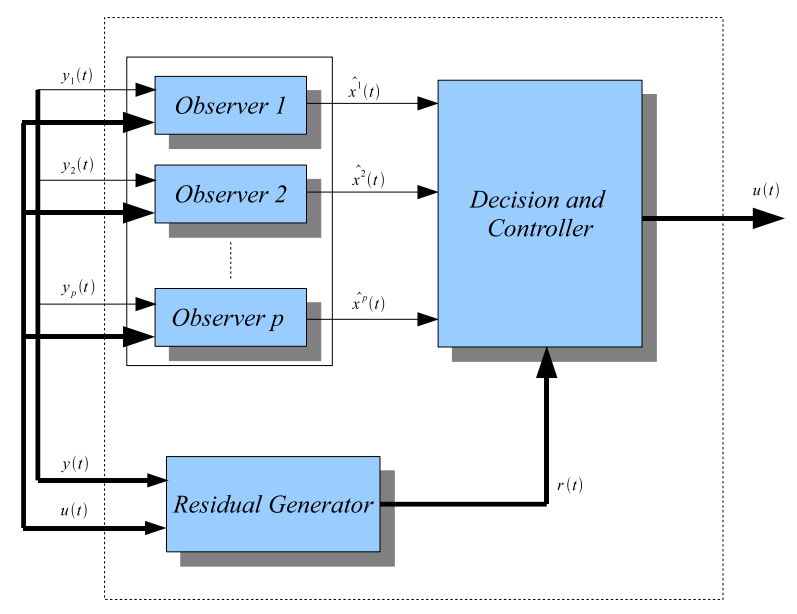

Fig. 1. Fault detection and fault tolerant control block

Its structure is the following:

$$
\left\{\begin{array}{l}
\dot{\hat{x}}^{k}(t)=\sum_{i=1}^{r} \mu_{i}(\xi(t))\left(A_{i} \hat{x}^{k}(t)+B_{i} u(t)+L_{i}^{k}\left(y^{k}(t)-\hat{y}^{k}(t)\right)\right) \\
\hat{y}^{k}(t)=\sum_{i=1}^{r} \mu_{i}(\xi(t)) C_{i}^{k} \hat{x}^{k}(t)
\end{array}\right.
$$

where $C_{i}^{k}$ is the $k^{t h}$ row of the matrix $C_{i}$ corresponding to the $k^{t h}$ sensor and $y^{k}(t)$, the $k^{t h}$ entry of the $y(t)$ vector.

So, the $k^{t h}$ observer provides the estimated state vector $\hat{x}^{k}(t)$ based on the knowledge of the input and the $k^{t h}$ output. The different state estimates $\hat{x}^{k}(t), k=1, \ldots, p$ are then blended to build a representative state estimate $\hat{x}_{b}(t)$ according to

$$
\hat{x}_{b}(t)=\sum_{k=1}^{p} h_{k}(r(t)) \hat{x}^{k}(t)
$$

The blending is ensured by the functions $h_{k}(r(t))$ depending on the residual vector $r(t)$ defined in (5). They are smooth nonlinear functions satisfying the convex sum property. The design of such functions is based on the idea that if the $k^{t h}$ sensor is affected by a fault, the $k^{t h}$ component of the residual, namely $r_{k}(t)$, is non zero. In this case, the function $h_{k}(r(t))$ must be close to zero in order to minimize the influence of $\hat{x}^{k}(t)$, which is affected by $f^{k}(t)$. In order to satisfy this property, it is proposed to define the functions $h_{k}$, for $k=1, \ldots, p$ as follows

$$
\begin{aligned}
\omega_{k}\left(r_{k}(t)\right) & =\exp \left(-r_{k}^{2}(t) / \sigma_{k}\right) \\
h_{k}(r(t)) & =\frac{\omega_{k}\left(r_{k}(t)\right)}{\sum_{\ell=1}^{p} \omega_{\ell}\left(r_{\ell}(t)\right)}
\end{aligned}
$$

where the parameters $\sigma_{k}$ are used to take into account the spreading of $r_{k}$ around zero. The Gaussian function (13a) causes an exponentially decreasing weight around zero. Equation (13b) ensures the standardization of the different functions such that they satisfy the convex sum property. With these definitions, a residual close to zero leads to a weight function tending to 1 whereas a residual significantly different from zero (in the sense of the variability $\sigma$ ) generates a weight tending to 0 .
The proposed control law is similar to a classical parallel distributed controller (PDC), but it is based on the knowledge of the "fault free" state estimate $\hat{x}_{b}(t)$

$$
u(t)=-\sum_{j=1}^{r} \mu_{j}(\xi(t)) K_{j} \hat{x}_{b}(t)
$$

Notice that in (Oudghiri et al., 2008), a bank of controllers is implemented, each of them is designed separately and generates a control law based on the state estimate $\hat{x}^{k}(t)$. Based on a residual analysis, a switching strategy is then developed in order to select "the best" control signal (in the sense that the control law relies on a fault free state estimate). Unfortunately, this strategy cannot guarantee the stability of the global system. As explained in (Liberzon and Morse, 1999), for switched systems, the stability of the local systems is a necessary but not sufficient condition to the stability of the global system. Whereas, with the proposed approach, the stability of the closed-loop system can be studied by using classical approaches developed for T-S models.

Let us now analyze the stability of the closed loop system. The $k^{t h}$ state estimation error $e^{k}(t)=x(t)-\hat{x}^{k}(t)$ is generated by the following differential equation

$$
\dot{e}^{k}(t)=\sum_{i=1}^{r} \sum_{j=1}^{r} \mu_{i}(\xi(t)) \mu_{j}(\xi(t))\left(A_{i}-L_{i}^{k} C_{j}^{k}\right) e^{k}(t)
$$

The closed-loop system is then described by

$$
\begin{aligned}
\dot{x}(t)= & \sum_{i=1}^{r} \sum_{j=1}^{r} \sum_{k=1}^{p} h_{k}(r(t)) \mu_{i}(\xi(t)) \mu_{j}(\xi(t))\left(B_{i} K_{j} e^{k}(t)\right. \\
& \left.+\left(A_{i}-B_{i} K_{j}\right) x(t)\right)
\end{aligned}
$$

Defining the augmented state vector

$$
x_{a}^{T}(t)=\left[x^{T}(t) e^{1 T}(t) \ldots e^{p T}(t)\right]
$$

the following closed-loop system is obtained

$$
\dot{x}_{a}(t)=\sum_{i=1}^{r} \sum_{j=1}^{r} \mu_{i}(\xi(t)) \mu_{j}(\xi(t))\left(\mathcal{A}_{i j}+\Delta \mathcal{A}_{i j}(t)\right) x_{a}(t)
$$

where

$$
\mathcal{A}_{i j}=\operatorname{diag}\left(A_{i}-B_{i} K_{j}, A_{i}-L_{i}^{1} C_{j}^{1}, \ldots, A_{i}-L_{i}^{p} C_{j}^{p}\right)
$$
and

$$
\Delta \mathcal{A}_{i j}(t)=\left[\begin{array}{ccccc}
0 & h_{1}(r) B_{i} K_{j} & h_{2}(r) B_{i} K_{j} & \ldots & h_{p}(r) B_{i} K_{j} \\
0 & 0 & 0 & \ldots & 0 \\
0 & 0 & 0 & \ddots & \vdots \\
\vdots & \vdots & \ddots & \ddots & 0 \\
0 & 0 & \ldots & 0 & 0
\end{array}\right]
$$

The observer and controller gains are obtained by solving the LMIs provided by the following theorem.

Theorem 5. Given the system (2) with $p$ sensors. Given a positive scalar $\eta$, the sensor fault tolerant observers based controller (11)-(14) ensures asymptotic stability of the system in the presence of sensor faults, if there exists symmetric and positive definite matrices $Q, P_{k}$ $(k=1, \ldots, p)$, matrices $F_{i}$ and $M_{i}^{k}$ and positive scalars $\epsilon$ and $\lambda_{k}$ such that the following LMI constraints hold for $i, j=1, \ldots, r$

$$
X_{i j}<0
$$


where

$$
\begin{aligned}
& X_{i j}=\left(\begin{array}{cc}
\mathcal{H}_{i j} & 0_{((2 p+1) n) \times((p+1) n)} \\
* & \mathcal{S}_{i j}
\end{array}\right) \\
& \mathcal{H}_{i j}=\left(\begin{array}{ccc}
\Xi_{i j} & \mathcal{R}_{i j} & 0_{n \times n p} \\
\mathcal{R}_{i j}^{T} & -2 \eta \tilde{Q} & \eta I_{n p \times n p} \\
0_{n p \times n} & \eta I_{n p \times n p} & \tilde{\Lambda}
\end{array}\right) \\
& \mathcal{S}_{i j}=\left(\begin{array}{cc}
\mathcal{M}_{i j} & 0_{n p \times n} \\
0_{n \times n p} & -\varepsilon I_{n}
\end{array}\right) \\
& \mathcal{M}_{i j}=\operatorname{diag}\left(\Delta_{i j}^{1}, \Delta_{i j}^{2}, \cdots, \Delta_{i j}^{p}\right) \\
& \mathcal{R}_{i j}=\left(\begin{array}{lll}
B_{i} F_{j} & \cdots & B_{i} F_{j}
\end{array}\right) \\
& \tilde{\Lambda}=\operatorname{diag}\left(-\lambda_{1} I_{n},-\lambda_{2} I_{n}, \ldots,-\lambda_{p} I_{n}\right) \\
& \tilde{Q}=\operatorname{diag}(Q, Q, \ldots, Q) \\
& \Xi_{i j}=Q A_{i}^{T}+A_{i} Q-B_{i} F_{j}-F_{j}^{T} B_{i}^{T} \\
& \Delta_{i j}^{k}=A_{i}^{T} P_{k}+P_{k} A_{i}-M_{i}^{k} C_{j}^{k}-\left(M_{i}^{k} C_{j}^{k}\right)^{T}+\lambda_{k} I_{n}
\end{aligned}
$$

The controller and observer gains are derived from

$$
K_{i}=F_{i} Q^{-1} \quad \text { and } \quad L_{i}^{k}=P_{k}^{-1} M_{i}^{k}
$$

Proof. Consider the quadratic Lyapunov function

$$
V\left(x_{a}(t)\right)=x_{a}^{T}(t) P x_{a}(t), \quad P=P^{T}>0
$$

where $P=\operatorname{diag}\left(X, P_{1}, \ldots, P_{p}\right)$. The time derivative of $V$ is given by

$$
\begin{aligned}
\dot{V}\left(x_{a}(t)\right) & =x_{a}^{T}(t) \sum_{i=1}^{r} \sum_{j=1}^{r} \mu_{i}(\xi(t)) \mu_{j}(\xi(t))\left(\mathcal{A}_{i j}^{T} P+P \mathcal{A}_{i j}\right. \\
& \left.+\Delta \mathcal{A}_{i j}^{T}(t) P+P \Delta \mathcal{A}_{i j}(t)\right) x_{a}(t)
\end{aligned}
$$

where $\Delta \mathcal{A}_{i j}(t)$ are time varying matrices given by

$$
\Delta \mathcal{A}_{i j}(t)=\underbrace{\left(\begin{array}{cccc}
0 & B_{i} K_{j} & \cdots & B_{i} K_{j} \\
0 & 0 & \ddots & \vdots \\
\vdots & \vdots & \ddots & 0 \\
0 & \cdots & 0 & 0
\end{array}\right)}_{\mathcal{K}_{i j}} \underbrace{\left(\begin{array}{cccc}
0 & 0 & \cdots & 0 \\
0 & h_{1}(r(t)) I & \cdots & \vdots \\
\vdots & \vdots & \ddots & \vdots \\
0 & \cdots & 0 & h_{p}(r(t)) I
\end{array}\right)}_{\Sigma(t)}
$$

Knowing that the functions $h_{k}(r(t))$ satisfy the convex sum property, it follows that $\Sigma^{T}(t) \Sigma(t) \leq \operatorname{diag}\left(0, I_{n}, \ldots, I_{n}\right)$. The derivative of the Lyapunov function is rewritten

$$
\begin{aligned}
\dot{V}\left(x_{a}(t)\right)= & x_{a}^{T}(t) \sum_{i=1}^{r} \sum_{j=1}^{r} \mu_{i}(\xi(t)) \mu_{j}(\xi(t))\left(\mathcal{A}_{i j}^{T} P+P \mathcal{A}_{i j}\right. \\
& \left.+\Sigma^{T}(t) \mathcal{K}_{i j}^{T} P+P \mathcal{K}_{i j} \Sigma(t)\right) x_{a}(t)
\end{aligned}
$$

Using the lemma 1 , one can bound $\dot{V}\left(x_{a}(t)\right)$ as follows

$$
\begin{aligned}
\dot{V}\left(x_{a}(t)\right) \leq & x_{a}^{T}(t) \sum_{i=1}^{r} \sum_{j=1}^{r} \mu_{i}(\xi(t)) \mu_{j}(\xi(t))\left(\mathcal{A}_{i j}^{T} P+P \mathcal{A}_{i j}\right. \\
& \left.+\Sigma^{T}(t) \Lambda \Sigma(t)+P \mathcal{K}_{i j} \Lambda^{-1} \mathcal{K}_{i j}^{T} P\right) x_{a}(t)
\end{aligned}
$$

where $\Lambda=\operatorname{diag}\left(\epsilon I_{n}, \lambda_{1} I_{n}, \lambda_{2} I_{n}, \ldots, \lambda_{p-1} I_{n}, \lambda_{p} I_{n}\right)$ is a block diagonal positive definite matrix. After calculation, the negativity of $\dot{V}\left(x_{a}(t)\right)$ is satisfied if

$$
\sum_{i=1}^{r} \sum_{j=1}^{r} \mu_{i}(\xi(t)) \mu_{j}(\xi(t)) Y_{i j}<0
$$

where $Y_{i j}$ is defined by

$$
Y_{i j}=\mathcal{A}_{i j}^{T} P+P \mathcal{A}_{i j}+\Sigma^{T} \Lambda \Sigma+P \mathcal{K}_{i j} \Lambda^{-1} \mathcal{K}_{i j}^{T} P
$$

The term $\Sigma^{T} \Lambda \Sigma$ can be bounded, by using the inequality $\Sigma^{T}(t) \Sigma(t) \leq \operatorname{diag}\left(0, I_{n}, \ldots, I_{n}\right)$, this leads to $\Sigma^{T} \Lambda \Sigma \leq \bar{\Lambda}$ where $\bar{\Lambda}=\overline{\operatorname{diag}}\left(0, \lambda_{1} I_{n}, \ldots, \lambda_{p} I_{n}\right)$. Due to the convex sum property of $\mu_{i}$, sufficient conditions satisfying (37) are $Y_{i j}<0, i, j=1, \ldots, r$. By applying the Schur complement (Boyd et al. (1994)), $Y_{i j}<0$ is equivalent to

$$
\left(\begin{array}{cc}
\mathcal{A}_{i j}^{T} P+P \mathcal{A}_{i j}+\bar{\Lambda} & P \mathcal{K}_{i j} \\
\mathcal{K}_{i j}^{T} P & -\Lambda
\end{array}\right)<0
$$

Using the congruence lemma 2 with the matrix

$$
W=\operatorname{diag}(X^{-1}, \underbrace{I_{n}, \cdots, I_{n}}_{p \text { matrices }}, \underbrace{X^{-1}, \cdots, X^{-1}}_{p \text { matrices }})
$$

and with the variable changes $Q=X^{-1}, F_{j}=K_{j} Q$ and $M_{i}^{k}=P_{k} L_{i}$, the following is obtained

$$
\left(\begin{array}{cccc}
\Xi_{i j} & 0_{n \times n p} & 0_{n \times n} & \mathcal{R}_{i j} \\
* & \mathcal{M}_{i j} & 0_{n \times n} & 0_{n p \times n p} \\
* & * & -\varepsilon I_{n} & 0_{n \times n p} \\
* & * & * & \tilde{Q} \tilde{\Lambda} \tilde{Q}
\end{array}\right)<0
$$

where $\Xi_{i j}, \mathcal{M}_{i j}, \mathcal{R}_{i j}, \tilde{Q}$ and $\tilde{\Lambda}$ are respectively defined in (29), (25), (26) (28) and (27). A nonlinearity lies in the last diagonal of the left hand term of the inequality (40), namely: $\tilde{Q} \tilde{\Lambda} \tilde{Q}$. From (21), $\tilde{\Lambda}$ is negative definite. Using lemma 3 , it follows that (40) is implied by

$$
\left(\begin{array}{cccc}
\Xi_{i j} & 0_{n \times n p} & 0_{n \times n} & \mathcal{R}_{i j} \\
* & \mathcal{M}_{i j} & 0_{n \times n} & 0_{n p \times n p} \\
* & * & -\varepsilon I_{n} & 0_{n \times n p} \\
* & * & * & -2 \eta \tilde{Q}-\eta^{2} \tilde{\Lambda}^{-1}
\end{array}\right)<0
$$

where $\eta$ is a positive scalar. With a Schur complement on the term $\eta^{2} \tilde{\Lambda}^{-1}$, it follows that (41) is equivalent to (21). Then (21) implies (40) and thus implies $\dot{V}(t)<0$, which achieves the proof.

Relaxed stability conditions The negativity of (37) is ensured if $X_{i j}<0, i, j=1, \ldots, r$. However, this result is conservative as often pointed in the literature. To overcome this limitation, one can use different methods of relaxations proposed recently as Tuan's lemma (Tuan et al., 2001) or Polya's theorem (Sala and Ariño, 2007) for example. In the following, the Polya's theorem is recalled and applied to the result proposed in theorem 5. Since $\left(\sum_{i=1}^{r} \mu_{i}(\xi(t))\right)^{q}=1$ where $q$ is any positive integer, the inequality (37) is equivalent to

$$
\left(\sum_{i=1}^{r} \mu_{i}(\xi(t))\right)^{q} \sum_{i=1}^{r} \sum_{j=1}^{r} \mu_{i}(\xi(t)) \mu_{j}(\xi(t)) X_{i j}<0
$$

Developing (42) in respect to the weighting functions, relaxed LMI conditions are obtained. Furthermore, if $q \rightarrow$ $\infty$ asymptotic necessary and sufficient conditions are obtained, as explained in Sala and Ariño (2007). For example, assuming $q=1$ the LMI constraints (21) are replaced by

$$
\begin{cases}X_{i i}<0, & i=1, \ldots, r \\ X_{i i}+X_{i j}+X_{j i}<0, & i, j=1, \ldots, r, \quad i \neq j\end{cases}
$$

\subsection{Algorithm of FTC design}

The design of the fault tolerant control law can be summarized by the following steps 
(1) Choose the filter $W_{\text {ref }}(s)$ and construct the residual generator (5) providing the residual signal $r(t)$ by solving the $\operatorname{LMI}(8)$, for $i, j=1, \ldots, r$.

(2) Construct the weighting functions $h_{k}(r(t))$ depending on the residual signal.

(3) Design of the FT controller, by solving the LMI (21) or $(43)$, for $i, j=1, \ldots, r$ and $k=1, \ldots, p$, where $K_{i}$ (resp. $L_{i}^{k}$ ) is substituted by $F_{i}\left(\operatorname{resp} . M_{i}^{k}\right)$.

\section{SIMULATION EXAMPLES}

To illustrate the proposed approach, let us consider the system (2), with $r=2$ submodels, defined by

$$
\begin{gathered}
A_{1}=\left(\begin{array}{ccc}
-2 & 1 & 1 \\
1 & -3 & 0 \\
2 & 1 & -8
\end{array}\right) \quad A_{2}=\left(\begin{array}{ccc}
-3 & 2 & -2 \\
5 & -3 & 0 \\
1 & 2 & -4
\end{array}\right) \\
B_{1}=\left(\begin{array}{c}
1 \\
5 \\
0.5
\end{array}\right) \quad B_{2}=\left(\begin{array}{c}
3 \\
1 \\
-1
\end{array}\right) \quad C_{1}=C_{2}=\left(\begin{array}{lll}
1 & 1 & 1 \\
1 & 0 & 0
\end{array}\right)
\end{gathered}
$$

The weighting functions are defined by

$$
\mu_{1}(y(t))=\frac{1-\tanh \left(y_{2}(t)\right)}{2}, \quad \mu_{2}(y(t))=1-\mu_{1}(y(t))
$$

A nonlinear observer based fault tolerant controller is designed by following the proposed procedure. Since there are two outputs, two state observers are built according to (11). A residual generator is also designed in order to generate the two signals detecting and isolating each sensor fault. Finally, the blending mechanism between the two state estimates is designed by defining the functions $h_{k}(r(t))$ such that $h_{k}(r(t))$ is close to zero when $f_{k}(t)$ occurs. This can be done using the functions $h_{k}$ defined in (13), with $\sigma_{1}=\sigma_{2}=0.01$. For the considered example, the controller given by (12) and (14) is designed. Different faults are considered in these simulations: the first ones are additive time varying faults and the second ones are parametric faults. For a comparison purpose, a classical observer based controller is designed according to Jadbabaie et al. (2000).

\subsection{Sensor additive time varying faults}

Let us consider two additive oscillatory faults, displayed on the top of figure 3. The first one is a low frequency fault affecting $y_{2}(t)$, while the second is a high frequency one affecting $y_{1}(t)$. With a classical PDC law, the state estimates are clearly very perturbed by the fault, especially in presence of the high frequency fault, whereas the proposed fault tolerant control law provides state estimation errors near zero. The figures 2 and 3 illustrate the results.

\subsection{Sensor parametric faults}

The second example considers a parametric fault, $f(t)$, and an additive fault, $f_{2}(t)$, simultaneously affecting a sensor. The faulty output $y_{1}(t)$ is as follows:

$$
y_{1}(t)=f(t) C^{1} x(t)+f_{2}(t)
$$

From $t=0$ to $t=22$, no fault is affecting the system output (i.e. $f(t)=1$ and $f_{2}(t)=0$ ). At the time instant $t=22$, the oscillatory fault $f(t)$ appears. In addition, a constant fault $f_{2}(t)$ with magnitude 1 affects the first sensor on the time interval $[30,32]$. These faults are depicted on the top of the figure 5 .
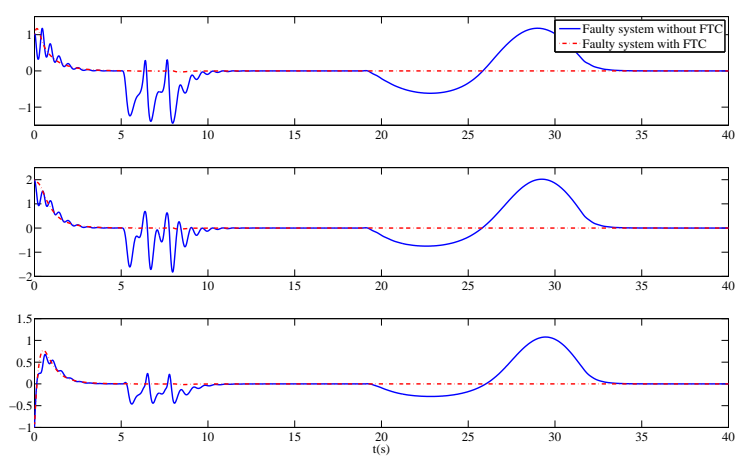

Fig. 2. System states with and without FTC
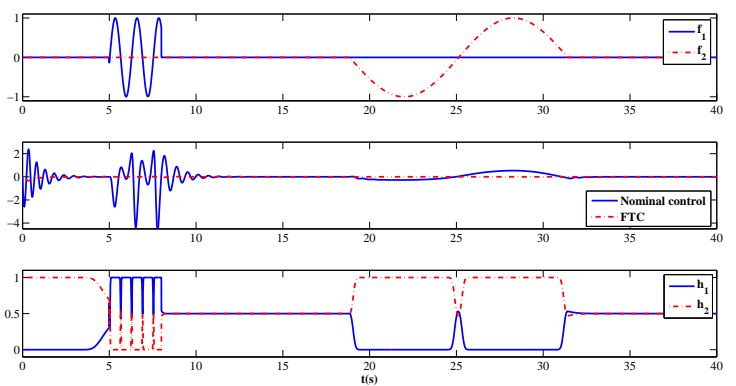

Fig. 3. Faults, control signals and weighting functions

Before $t=22$, without any fault affecting the system, the controlled systems with and without FTC have the same response. It is due to the fact that at time instant $t=22$ in the fault free case, the system is stabilized and then the system states have converged towards zero. But, if any small perturbation occurs on any component of the system (sensors, actuators,...) the system state deviates from zero and the system without FTC may become unstable according to the type of the parametric fault. The system controlled with FTC remains stable and the state trajectories have a very small deviation from zero. This latter is corrected by the fault tolerant controller, whereas the system controlled by the classical approach becomes unstable, as shown in the figures 4 and 5 .
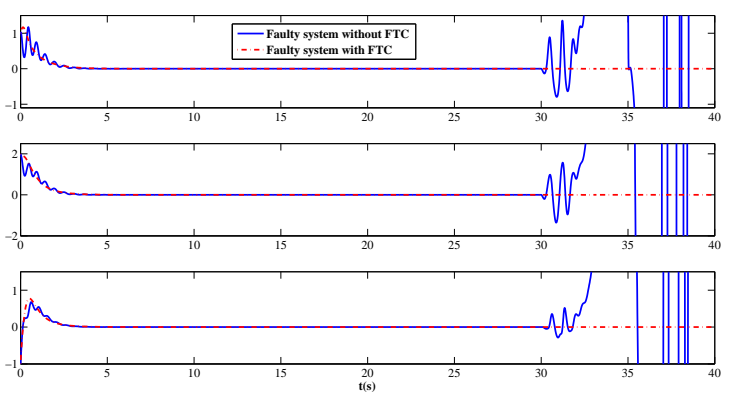

Fig. 4. System states with and without FTC

\section{CONCLUSIONS}

In this paper, a new approach is proposed to design a sensor fault tolerant controller for nonlinear complex systems represented by T-S model. The approach is based on a 

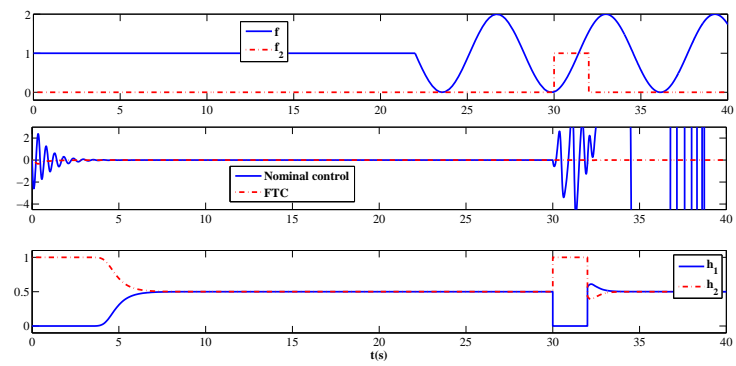

Fig. 5. Faults, control signals and weighting functions

bank of observers-based controllers, a residual generator for diagnosis and a smooth selecting mechanism to choose an adequate state estimate to compensate the effects of the faults on the system. The stability of the whole system is studied by Lyapunov theory and LMI constraints are provided to design the gain matrices of the different blocks of the proposed FTC scheme. For future works, it will be interesting to consider the case of T-S systems with unmeasurable premise variables. It would also be interesting to study the choice of the functions $h_{k}(r(t))$. Finally, the dedicated scheme may be inapplicable in some cases since the system state needs to be reconstructed based on each output. Consequently the proposed strategy could be extended using a Generalized Observer Scheme.

\section{REFERENCES}

Akhenak, A., Chadli, M., Ragot, J., and Maquin, D. (2008). Fault detection and isolation using sliding mode observer for uncertain Takagi-Sugeno fuzzy model. In 16th Mediterranean Conference on Control and Automation. Ajaccio, France.

Bergsten, P., Palm, R., and Driankov, D. (2002). Observers for Takagi-Sugeno fuzzy systems. IEEE Transactions on Systems, Man, and Cybernetics - Part B: Cybernetics, 32(1), 114-121.

Boyd, S., El Ghaoui, L., Feron, E., and Balakrishnan, V. (1994). Linear Matrix Inequalities in System and Control Theory. SIAM ed.

Chen, W. and Saif, M. (2007). Design of a TS based fuzzy nonlinear unknown input observer with fault diagnosis applications. In American Control Conference. New York City, USA.

Ding, S.X. (2008). Model-Based Fault Diagnosis Techniques Design Schemes, Algorithms, And Tools. Springer-Verlag.

Gasso, G., Mourot, G., and Ragot, J. (2002). Environmental systems modelling and diagnosis using a multiple model approach. In 5th International FLINS Conference on Computational Intelligent Systems for Applied Research. Gent, Belgium.

Guerra, T.M., Kruszewski, A., Vermeiren, L., and Tirmant, H. (2006). Conditions of output stabilization for nonlinear models in the Takagi-Sugeno's form. Fuzzy Sets and Systems, 157(9), 1248-1259.

Ichalal, D., Marx, B., Ragot, J., and Maquin, D. (2009a). An approach for the state estimation of Takagi-Sugeno models and application to sensor fault diagnosis. In 48th IEEE Conference on Decision and Control, CDC'09. Shanghai, P.R. China.
Ichalal, D., Marx, B., Ragot, J., and Maquin, D. (2009b). Fault diagnosis in Takagi-Sugeno nonlinear systems. In 7th IFAC Symposium on Fault Detection, Supervision and Safety of Technical Processes, SAFEPROCESS'2009. Barcelona, Spain.

Ichalal, D., Marx, B., Ragot, J., and Maquin, D. (2010). Fault tolerant control for Takagi-Sugeno systems with unmeasurable premise variables by trajectory tracking. In IEEE International workshop on Industrial Electronics, ISIE'10. Bari, Italy.

Jadbabaie, A., Abdallah, T., Jamshidi, M., and Titli, A. (2000). Observer-based controller synthesis for modelbased fuzzy systems via linear matrix inequalities. Fuzzy Control Synthesis and analysis, 239-252.

Kruzewski, A., Wang, R., and Guerra, T. (2008). Nonquadratic stabilization conditions for a class of uncertain non linear discrete-time T-S fuzzy models: a new approach. IEEE Transactions on Automatic Control, $53(2), 606-611$.

Liberzon, D. and Morse, A.S. (1999). Basic problems in stability and design of switched systems. IEEE Control Systems Magazine, 19(5), 59-70.

Marx, B., Koenig, D., and Ragot, J. (2007). Design of observers for Takagi Sugeno descriptor systems with unknown inputs and application to fault diagnosis. IET Control Theory and Application, 1(5), 1487-1495.

Oudghiri, M., Chadli, M., and El Hajjaji, A. (2008). Robust observer-based fault tolerant control for vehicle lateral dynamics. International Journal of Vehicle Design, 48(3-4), 173-189.

Sala, A. and Ariño, C. (2007). Asymptotically necessary and sufficient conditions for stability and performance in fuzzy control: Applications of Polya's theorem. Fuzzy Sets and Systems, 158(24), 2671-2686.

Takagi, T. and Sugeno, M. (1985). Fuzzy identification of systems and its applications to modeling and control. IEEE Transactions on Systems, Man, and Cybernetics, 15(1), 116-132.

Tanaka, K., Hori, T., and Wang, H. (2003). A multiple Lyapunov function approach to stabilization of fuzzy control systems. IEEE Transactions on Fuzzy Systems, 11(4), 582- 589.

Tanaka, K. and Wang, H. (2001). Fuzzy Control Systems Design and Analysis: A Linear Matrix Inequality Approach. John Wiley and Sons.

Tuan, H., Apkarian, P., Narikiyo, T., and Yamamoto, Y. (2001). Parameterized linear matrix inequality techniques in fuzzy control system design. IEEE Transactions on Fuzzy Systems, 9(2), 324-332. 